\title{
Relation between air pollutants emissions and BESS capacity operated for maximizing customer's profit
}

\author{
Woo-Cheol Jeong ${ }^{1,}{ }^{*}$, Myung-Su Kim ${ }^{2}$, Jong-Bae Park ${ }^{3}$, Jae Hyung Roh ${ }^{4}$ \\ 1, 2, 3, 4 Department of Electrical Engineering, Konkuk University, Seoul 05029, Korea
}

\author{
Index Terms \\ Relation \\ GHGs Emission \\ Power \\ BESS \\ Unit Commitment
}

Received: 5 August 2015

Accepted: 23 September 2015

Published: 24 June 2016

\begin{abstract}
At COP 21, participants of the UNFCC reached an agreement for mitigation of greenhouse gas emissions. It is not mandatory, but new policies and technologies are needed for each country to accomplish Intended Nationally Determined Contributions (INDC). Especially in the power section, Smart Grid, Renewable energy, Battery Energy Storage System (BESS), Distributed Generation and Microgrid are emerged as solutions to reduce GHGs. These technologies are known as the GHGs mitigation Technologies. However, researches are needed to reveal that is true or not. BESS can be operated for diverse purpose. This paper presents the GHGs emissions changes resulting from the unit commitment with BESS that is applied to Korea power system for minimizing end-user's costs. This study is based on IEEE 39-bus system to reveal the influence of BESS on GHGs. As a result, in a certain condition, the increasing of BESS capacity could result the increasing of GHGs emissions.
\end{abstract}

\section{INTRODUCTION}

The Kyoto Protocol which put obligation to reduce greenhouse gas emissions on developed countries was limited to curb climate change. So, new framework that can reduce risks and impacts of climate change was needed. As a result, 'Paris agreement' was adopted by consensus at 21st Conference of the Parties of the UNFCCC on Dec 2015.

Each country should accomplish their Intended Nationally Determined Contributions, but they are not bound by the international laws. The only duty of member nations is that contributions should be reported to UNFCCC secretariat every five years. Despite the circumstances,
(C) 2016 The Author(s). Published by TAF Publishing.

\footnotetext{
*Corresponding author: Woo-Cheol Jeong

E-mail: ganrf@naver.com
}

new policies and technologies like energy savings, improving energy efficiency, renewable generation are needed to accomplish INDC. Especially in the power section, Smart Grid, Renewable energy, BESS, Distributed Generation and Micro-grid are emerged as solutions to reduce GHGs.

These technologies are also known as the GHGs mitigation Technologies. If they are commercialized and restructuring of power market is done, customers who will live in smart cities will be able to participate in power trading, and they try to set a schedule for minimizing their electricity usage cost by using advanced technology. [1] This paper focuses on relationship between GHGs emission and these activities of customers who have BESS and operating it. 


\section{ENERGY STORAGE SYSTEM}

\section{A. Definition and Classification}

ESS can overcome electricity's essential characteristic that production and consumption happen at the same time and they must be balanced. It can make using power more efficient. Generally grid-scale(for transmission, distribution) ESS can be used in a variety of ways - load leveling, peak cutting, frequency regulation, solution to renewable energy's intermittency, and emergency reserve. When customers use small-scale (up to $10 \mathrm{MW}$ ) ESS, they would get benefit from difference in energy price. Or they could make profit by operating ESS with renewable generation. Customers who live in several countries that have already reached grid parity combine renewable generation and ESS, and then consume power themselves or sell surplus power to make their profits. The others who are not living in where grid parity isn't reached are operating ESS to save their power rates, or get margin by charging during off-peak and discharging on peak.

\section{B. Current State of BESS in Korea}

Korea government chose Frequency Regulation BESS as the one of 'new energy industry project'. They have installed 236 MW till 2015, and make plans to expand capacity up to $500 \mathrm{MW}$ and improve performance. The Laws and regulations were revised to allow ESS to use it as emergency power source. If ESS capacity is larger than 1 MW, its owner can participate in power market. Also, large capacity ESS which is more than $10 \mathrm{MW}$ is considered as scheduled generator that means generator dispatched by system operator-Korea Power eXchange(KPX). Government adopted Renewable standard portfolio which is a regulatory mandate to increase production of energy from renewable sources since 2012. If renewable providers interconnect their existing solar with ESS, they could get more renewable energy certificates (REC) than their real generation quantity. Also, government made a new electricity pricing rate which will save base rate as much as customer's reduced maximum peak to promote investment for ESS by shortening investment payback period [2].

TABLE 1

F/R ESS CONSTRUCTION SITUATION AND PLAN [2]

\begin{tabular}{llllll}
\hline \hline Year & 2014 & 2015 & 2016 & 2017 & Total \\
\hline $\begin{array}{l}\text { Capacity(MW) } \\
\text { Budget(100 million }\end{array}$ & 52 & 184 & 140 & 124 & 500 \\
KRW) & 570 & 1,900 & 1,890 & 1,890 & 6,250 \\
\hline \hline
\end{tabular}

TABLE 2

CURRENT SITUATION OF BESS CONSTRUCTION IN KOREA OF 2015 [2]

\begin{tabular}{|c|c|c|c|c|c|c|c|c|}
\hline S/S location & $\begin{array}{l}\text { New } \\
\text { Gyeryong }\end{array}$ & $\begin{array}{l}\text { New } \\
\text { Gimje }\end{array}$ & $\begin{array}{l}\text { New } \\
\text { Hwasun }\end{array}$ & Ulju & Uiryeong & Gyeongsan & $\begin{array}{l}\text { New } \\
\text { Chungju }\end{array}$ & Total \\
\hline capacity(MW) & 24 & 24 & 24 & 24 & 24 & 48 & 16 & 184 \\
\hline
\end{tabular}

\section{Power system of Korea}

Base load is the minimum level of electricity demand required over a period of 24 hours. It is needed to provide enough power to components that keep running at all times. Peak load is the time of high demand. Nuclear and coal-fired plants are base load plants, and the others like LNG, diesel plants which use more expensive fuels than base load plants are used for peak load in Korea. When customers use ESS for minimizing their power cost, they would charge ESS in base time when electricity price is low and discharge power to grid in peak time when the price is expensive, if ESS is commercialized, and result of power market restructuring makes power price as Real time price. Customer's activities as above would reduce daily maximum peak load and increase load factor, so these also reduce total energy production cost of power system. It is widely known that coal-fired plants emit GHGs more than LNG plants. The fact that the spread of ESS can reduce GHGs emission is seen as a true, but it may well be wrong because ESS operation for cost minimization will shift energy at peak time to off-peak when base load plants operate. Nuclear plants are already operated at maximum power output, so coal-fired must be used to handle shifted energy 


\section{METHODOLOGY}

Study on entire power system in Korea has lots of difficulties because of scale and complexity of problem. So IEEE 39-bus system data is used for case study in this paper. We set 4 cases. The first is case without ESS, and the others are applying different ESS capacity.

TABLE 3

ESS CAPACITY PER EACH SCENARIO

\begin{tabular}{l|l}
\hline \hline Scenario & ESS capacity (MW) \\
\hline 1 & None \\
2 & 100 \\
3 & 200 \\
4 & 300 \\
\hline \hline
\end{tabular}

And we determine Unit Commitment in each case, and calculate total generation cost, $\mathrm{CO}_{2}, \mathrm{NOx}$ and $\mathrm{SOx}$ emissions, health cost and treatment cost. Table 4 shows us air pollutants emission factors in electric power plants. Processing cost is shown in table 5. In this paper, ESS is modeled as load shifting resource and affects result of unit commitment by changing daily load. This paper formulates unit commitment algorithm based on dynamic programing and compares results from previous papers to verify accuracy.

TABLE 4

AIR POLLUTANTS EMISSION FACTORS (TON/MWH) [3]

\begin{tabular}{c|c|c|c}
\hline \hline Pollutant & Anthracite & Bituminous & LNG \\
\hline $\mathrm{CO}_{2}$ & 0.9143 & 0.823 & 0.3625 \\
$\mathrm{SOx}$ & 0.00061 & 0.00039 & 0.00000 \\
$\mathrm{NOx}$ & 0.00098 & 0.00032 & 0.00003 \\
\hline \hline
\end{tabular}

TABLE 5

PROCESSING COST [4], [5]

Pollutant Cost (KRW/ton)

$\begin{array}{ll}\text { CO2 } & 21,000 \\ \text { SOx } & 16,050,000 \\ \text { NOx } & 11,400,000\end{array}$

\section{A. Modeling of load-shifting operation of ESS [6]}

The objective function for load-shifting is formulated as below.

Minmize $\sum_{t=1}^{T}\left(L_{t}+P_{t}^{E S S}\right)^{2}$

$P_{t}^{E S S}=e p_{t}^{c}-e p_{t}^{d}$

There are some technical constraints for operating ESS. $E P_{\mathrm{t}}^{\mathrm{c}}, E P_{t}^{d}$ are charging power and discharging power (MW) inside view on ESS at time $t$. These are formulated by using efficiency constants and charging power and discharging power in view of power system at time $t$. This paper assumes that there are no losses, so efficiency constants are 1 , because we just need to check effect of shifted power and changing in pollutants from fluctuation of each generator's output.

$$
\mathrm{EP}_{\mathrm{t}}^{\mathrm{c}}=\eta^{c} \times e p_{t}^{c}
$$

$\mathrm{EP}_{\mathrm{t}}^{\mathrm{d}}=e p_{t}^{d} / \eta^{d}$

(Constraint (5) imposes a limit on the charging and discharging power of the ESS.

$0 \leq \mathrm{EP}_{\mathrm{t}}^{\mathrm{c}} \leq \mathrm{EP}^{\text {rated }}, 0 \leq \mathrm{EP}_{\mathrm{t}}^{\mathrm{d}} \leq \mathrm{EP}^{\text {rated }}$

Eq. (6) describes the SOC of ESS. Constraint (6) limits SOC of the battery to be less than ESS capacity and prevents the deep discharging from ESS by imposing minimum limit of SOC.

$\mathrm{SOC}^{\text {min }} \leq S O C^{\text {initial }}+\sum_{i=1}^{t} E P_{i}^{c}-\sum_{i=1}^{t} E P_{i}^{d} \leq S O C^{\text {max }}$,

each $\forall t$

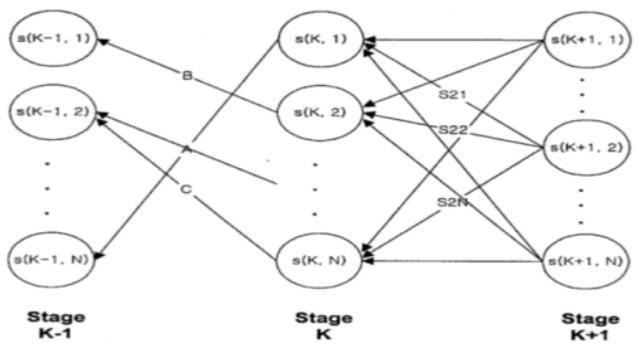

Fig. 1. states of dynamic programming

Eq. (7) is final condition limit of SOC at end time T. SOC at time T must be between $S O C^{\text {final1 }}$ and $S O C^{\text {final2 }}$.

$$
\mathrm{SOC}^{\text {final1 }} \leq S O C^{\text {initial }}+\sum_{i=1}^{T} E P_{i}^{c}-\sum_{i=1}^{T} E P_{i}^{d} \leq
$$
SOC final2 (7)

$E P^{\text {rated }}$ : rated power of ESS (MW)

$\eta^{c}, \eta^{d}$ : charging efficiency and discharging efficiency of ESS.

$\mathrm{SOC}_{\mathrm{t}}$ : SOC of ESS at time t. (MWh)

SOC ${ }^{\text {initial }}$ : Initial SOC of ESS (MWh)

SOC $^{\text {final }}$ : Final SOC of ESS (MWh) 
SOC $^{\text {min }}$ : Minimum allowed SOC of ESS (MWh)

SOC $^{\text {max }}$ : Maximum allowed SOC of ESS (MWh)

This paper uses forward dynamic programming methods for Unit Commitment. Figure 1 shows the concept of DP. Table 6 is basic load data of case1. Table 7 shows specs of each generator-fuel, power out range, constants of cost function $(a, b, c)$, minimum down time, minimum up time and etc.

TABLE 7

GENERATING UNIT DATA

\begin{tabular}{|c|c|c|c|c|c|c|c|c|c|c|}
\hline Unit & Unit1 & Unit2 & Unit3 & Unit4 & Unit5 & Unit6 & Unit7 & Unit8 & Unit9 & $\begin{array}{c}\text { Unit1 } \\
0\end{array}$ \\
\hline fuel & $\begin{array}{c}\text { nuclea } \\
\mathrm{r}\end{array}$ & $\begin{array}{c}\text { nuclea } \\
\mathrm{r}\end{array}$ & $\begin{array}{c}\text { anthracit } \\
\mathrm{e}\end{array}$ & $\begin{array}{c}\text { anthracit } \\
\mathrm{e}\end{array}$ & $\begin{array}{c}\text { anthracit } \\
\mathrm{e}\end{array}$ & $\begin{array}{c}\text { bituminou } \\
\mathrm{s}\end{array}$ & $\begin{array}{c}\text { bituminou } \\
\mathrm{s}\end{array}$ & LNG & LNG & LNG \\
\hline $\begin{array}{l}\text { Min } \\
(\mathrm{MW})\end{array}$ & 150 & 150 & 20 & 20 & 25 & 20 & 25 & 10 & 10 & 10 \\
\hline $\begin{array}{c}\text { Max } \\
(\mathrm{MW})\end{array}$ & 455 & 455 & 130 & 130 & 162 & 80 & 85 & 55 & 55 & 55 \\
\hline$a_{1}$ & 0.00048 & 0.00031 & 0.002 & 0.00211 & 0.00398 & 0.00712 & 0.00079 & $\begin{array}{c}0.0041 \\
3\end{array}$ & $\begin{array}{c}0.0022 \\
2\end{array}$ & 0.00173 \\
\hline$b_{1}$ & 16.19 & 17.26 & 16.60 & 16.50 & 19.70 & 22.26 & 27.74 & 25.92 & 27.72 & 27.79 \\
\hline$c_{1}$ & 1000 & 970 & 700 & 680 & 450 & 370 & 480 & 660 & 665 & 670 \\
\hline $\begin{array}{l}\text { MUT } \\
(\mathrm{H})\end{array}$ & 8 & 8 & 5 & 5 & 6 & 3 & 3 & 1 & 1 & 1 \\
\hline $\begin{array}{l}\text { MDT } \\
(\mathrm{H})\end{array}$ & 8 & 8 & 5 & 5 & 6 & 3 & 3 & 1 & 1 & 1 \\
\hline $\begin{array}{l}\text { Cold } \\
\text { Start } \\
\text { Time }\end{array}$ & 5 & 5 & 4 & 4 & 4 & 2 & 2 & 0 & 0 & 0 \\
\hline $\begin{array}{c}\text { Hot Start } \\
\text { Cost } \\
(\$)\end{array}$ & 4500 & 5000 & 550 & 560 & 900 & 170 & 260 & 30 & 30 & 30 \\
\hline $\begin{array}{c}\text { Cold } \\
\text { Start } \\
\text { Cost } \\
(\$)\end{array}$ & 9000 & 10000 & 1100 & 1120 & 1800 & 340 & 520 & 60 & 60 & 60 \\
\hline $\begin{array}{c}\text { Initial } \\
\text { Status(H } \\
\text { ) }\end{array}$ & 8 & 8 & -5 & -5 & -6 & -3 & -3 & -1 & -1 & -1 \\
\hline
\end{tabular}




\section{RESULTS}

TABLE 8

LOAD CHANGING RESULT OF EACH CAPACITY

\begin{tabular}{|c|c|c|c|c|c|c|c|c|}
\hline & 1 & 2 & 3 & 4 & 5 & 6 & 7 & 8 \\
\hline ESS 0 & 700 & 750 & 850 & 950 & 1000 & 1100 & 1150 & 1200 \\
\hline ESS 100 & 724.998 & 775 & 950 & 950 & 999.993 & 1100 & 1150 & 1200 \\
\hline ESS 200 & 750 & 800 & 850 & 950 & 1000 & 1100 & 1150 & 1200 \\
\hline \multirow[t]{2}{*}{ ESS 300} & 775 & 825 & 850 & 950 & 1000 & 1100 & 1150 & 1200 \\
\hline & 9 & 10 & 11 & 12 & 13 & 14 & 15 & 16 \\
\hline ESS 0 & 1300 & 1400 & 1450 & 1500 & 1400 & 1300 & 1200 & 1050 \\
\hline ESS 100 & 1300 & 1400 & 1449.833 & 1450.169 & 1400 & 1300 & 1200 & 1050.37 \\
\hline ESS 200 & 1300 & 1400 & 1425 & 1425 & 1400 & 1300 & 1200 & 1075 \\
\hline \multirow[t]{2}{*}{ ESS 300} & 1300 & 1400 & 1400 & 1400 & 1400 & 1300 & 1200 & 1100 \\
\hline & 17 & 18 & 19 & 20 & 21 & 22 & 23 & 24 \\
\hline ESS 0 & 1000 & 1100 & 1200 & 1400 & 1300 & 1100 & 900 & 800 \\
\hline ESS 100 & 1049.628 & 1100 & 1200 & 1350 & 1299.996 & 1100 & 900 & 799.999 \\
\hline ESS 200 & 1075 & 1100 & 1200 & 1300 & 1299.995 & 1100 & 900 & 800 \\
\hline ESS 300 & 1100 & 1100 & 1200 & 1275 & 1275 & 1100 & 900 & 800 \\
\hline
\end{tabular}

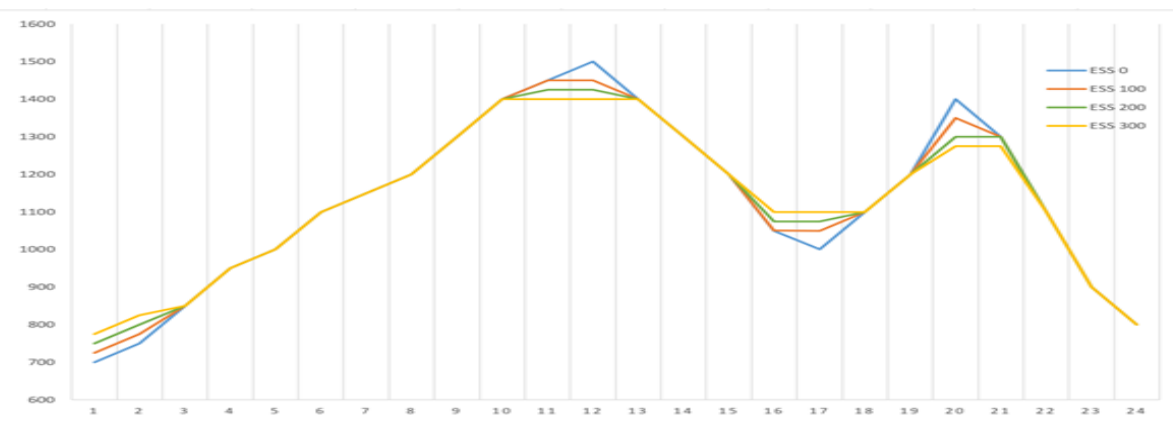

Fig. 2. Load changing result of each capacity 
TABLE 9

UNIT COMMITMENT RESULT OF SCENARIO 1

\begin{tabular}{|c|c|c|c|c|c|c|c|c|c|c|c|c|}
\hline \multirow{2}{*}{$\mathrm{hr}$} & \multirow[b]{2}{*}{ Unit 1} & \multirow[b]{2}{*}{ Unit 2} & \multirow[b]{2}{*}{ Unit 3} & \multicolumn{4}{|c|}{ Power output (MW) } & \multirow[b]{2}{*}{ Unit 8} & \multirow[b]{2}{*}{ Unit 9} & \multirow[b]{2}{*}{ Unit 10} & \multirow{2}{*}{$\begin{array}{l}\text { Total } \\
\text { power } \\
(\mathrm{MW})\end{array}$} & \multirow{2}{*}{$\begin{array}{c}\text { Cost } \\
(\$)\end{array}$} \\
\hline & & & & Unit 4 & Unit 5 & Unit 6 & Unit 7 & & & & & \\
\hline 1 & 455 & 245 & 0 & 0 & 0 & 0 & 0 & 0 & 0 & 0 & 700 & 13683.13 \\
\hline 2 & 455 & 295 & 0 & 0 & 0 & 0 & 0 & 0 & 0 & 0 & 750 & 14554.50 \\
\hline 3 & 455 & 395 & 0 & 0 & 0 & 0 & 0 & 0 & 0 & 0 & 850 & 16301.89 \\
\hline 4 & 455 & 455 & 0 & 0 & 40 & 0 & 0 & 0 & 0 & 0 & 950 & 18597.67 \\
\hline 5 & 455 & 455 & 0 & 0 & 90 & 0 & 0 & 0 & 0 & 0 & 1000 & 19608.54 \\
\hline 6 & 455 & 455 & 130 & 0 & 60 & 0 & 0 & 0 & 0 & 0 & 1100 & 21891.43 \\
\hline 7 & 455 & 455 & 130 & 0 & 110 & 0 & 0 & 0 & 0 & 0 & 1150 & 22910.26 \\
\hline 8 & 455 & 455 & 130 & 0 & 160 & 0 & 0 & 0 & 0 & 0 & 1200 & 23948.99 \\
\hline 9 & 455 & 455 & 130 & 130 & 130 & 0 & 0 & 0 & 0 & 0 & 1300 & 26184.02 \\
\hline 10 & 455 & 455 & 130 & 130 & 162 & 68 & 0 & 0 & 0 & 0 & 1400 & 28768.21 \\
\hline 11 & 455 & 455 & 130 & 130 & 162 & 63 & 0 & 0 & 55 & 0 & 1450 & 30848.56 \\
\hline 12 & 455 & 455 & 130 & 130 & 162 & 78 & 25 & 10 & 55 & 0 & 1500 & 33291.13 \\
\hline 13 & 455 & 455 & 130 & 130 & 162 & 68 & 0 & 0 & 0 & 0 & 1400 & 28768.21 \\
\hline 14 & 455 & 455 & 130 & 130 & 130 & 0 & 0 & 0 & 0 & 0 & 1300 & 26184.02 \\
\hline 15 & 455 & 455 & 130 & 0 & 160 & 0 & 0 & 0 & 0 & 0 & 1200 & 23948.99 \\
\hline 16 & 455 & 440 & 130 & 0 & 25 & 0 & 0 & 0 & 0 & 0 & 1050 & 20927.03 \\
\hline 17 & 455 & 390 & 130 & 0 & 25 & 0 & 0 & 0 & 0 & 0 & 1000 & 20051.16 \\
\hline 18 & 455 & 455 & 130 & 0 & 25 & 0 & 0 & 10 & 25 & 0 & 1100 & 23469.09 \\
\hline 19 & 455 & 455 & 130 & 0 & 50 & 20 & 25 & 10 & 55 & 0 & 1200 & 26798.02 \\
\hline 20 & 455 & 455 & 130 & 0 & 162 & 70 & 63 & 10 & 55 & 0 & 1400 & 31300.72 \\
\hline 21 & 455 & 455 & 130 & 130 & 130 & 0 & 0 & 0 & 0 & 0 & 1300 & 26184.02 \\
\hline 22 & 455 & 455 & 0 & 130 & 60 & 0 & 0 & 0 & 0 & 0 & 1100 & 21860.29 \\
\hline 23 & 455 & 315 & 0 & 130 & 0 & 0 & 0 & 0 & 0 & 0 & 900 & 17764.14 \\
\hline 24 & 455 & 215 & 0 & 130 & 0 & 0 & 0 & 0 & 0 & 0 & 800 & 16021.71 \\
\hline
\end{tabular}


TABLE10

UNIT COMMITMENT RESULT OF SCENARIO 2

\begin{tabular}{|c|c|c|c|c|c|c|c|c|c|c|c|c|}
\hline \multirow{2}{*}{$\mathrm{hr}$} & \multirow[b]{2}{*}{ Unit 1} & \multirow[b]{2}{*}{ Unit 2} & \multirow[b]{2}{*}{ Unit 3} & \multicolumn{4}{|c|}{ Power output (MW) } & \multirow[b]{2}{*}{ Unit 8} & \multirow[b]{2}{*}{ Unit 9} & \multirow[b]{2}{*}{ Unit 10} & \multirow{2}{*}{$\begin{array}{l}\text { Total } \\
\text { power } \\
(\mathrm{MW})\end{array}$} & \multirow{2}{*}{$\begin{array}{c}\text { Cost } \\
(\$)\end{array}$} \\
\hline & & & & Unit 4 & Unit 5 & Unit 6 & Unit 7 & & & & & \\
\hline 1 & 455 & 270 & 0 & 0 & 0 & 0 & 0 & 0 & 0 & 0 & 724.998 & 14118.59 \\
\hline 2 & 455 & 320.01 & 0 & 0 & 0 & 0 & 0 & 0 & 0 & 0 & 775.006 & 14990.87 \\
\hline 3 & 455 & 395 & 0 & 0 & 0 & 0 & 0 & 0 & 0 & 0 & 850 & 16301.89 \\
\hline 4 & 455 & 455 & 0 & 0 & 40 & 0 & 0 & 0 & 0 & 0 & 950 & 18597.67 \\
\hline 5 & 455 & 455 & 0 & 0 & 89.993 & 0 & 0 & 0 & 0 & 0 & 999.9931 & 19608.40 \\
\hline 6 & 455 & 455 & 130 & 0 & 60.007 & 0 & 0 & 0 & 0 & 0 & 1100.007 & 21891.57 \\
\hline 7 & 455 & 455 & 130 & 0 & 110 & 0 & 0 & 0 & 0 & 0 & 1150 & 22910.26 \\
\hline 8 & 455 & 455 & 130 & 0 & 160 & 0 & 0 & 0 & 0 & 0 & 1200 & 23948.99 \\
\hline 9 & 455 & 455 & 130 & 130 & 130 & 0 & 0 & 0 & 0 & 0 & 1300 & 26184.02 \\
\hline 10 & 455 & 455 & 130 & 130 & 162 & 68 & 0 & 0 & 0 & 0 & 1400 & 28768.21 \\
\hline 11 & 455 & 455 & 130 & 130 & 162 & 62.833 & 0 & 0 & 55 & 0 & 1449.833 & 30844.70 \\
\hline 12 & 455 & 455 & 130 & 130 & 162 & 38.169 & 25 & 0 & 55 & 0 & 1450.169 & 31451.93 \\
\hline 13 & 455 & 455 & 130 & 130 & 162 & 0 & 25 & 0 & 43 & 0 & 1400 & 29886.67 \\
\hline 14 & 455 & 455 & 130 & 130 & 105 & 0 & 25 & 0 & 0 & 0 & 1300 & 26842.13 \\
\hline 15 & 455 & 455 & 130 & 0 & 160 & 0 & 0 & 0 & 0 & 0 & 1200 & 23948.99 \\
\hline 16 & 455 & 440.37 & 130 & 0 & 25 & 0 & 0 & 0 & 0 & 0 & 1050.37 & 20933.51 \\
\hline 17 & 455 & 439.63 & 130 & 0 & 25 & 0 & 0 & 0 & 0 & 0 & 1049.628 & 20920.50 \\
\hline 18 & 455 & 420 & 130 & 0 & 40 & 20 & 25 & 10 & 0 & 0 & 1100 & 23787.53 \\
\hline 19 & 455 & 455 & 130 & 0 & 115 & 20 & 25 & 0 & 0 & 0 & 1200 & 25005.28 \\
\hline 20 & 455 & 455 & 130 & 0 & 162 & 80 & 58.006 & 10 & 0 & 0 & 1350.006 & 29198.68 \\
\hline 21 & 455 & 435 & 130 & 130 & 105 & 20 & 25 & 0 & 0 & 0 & 1299.996 & 27309.38 \\
\hline 22 & 455 & 435 & 0 & 130 & 60 & 20 & 0 & 0 & 0 & 0 & 1100 & 22327.62 \\
\hline 23 & 455 & 315 & 0 & 130 & 0 & 0 & 0 & 0 & 0 & 0 & 900 & 17764.14 \\
\hline 24 & 455 & 215 & 0 & 130 & 0 & 0 & 0 & 0 & 0 & 0 & 799.9994 & 16021.70 \\
\hline
\end{tabular}


TABLE 11

UNIT COMMITMENT RESULT OF SCENARIO 3

\begin{tabular}{|c|c|c|c|c|c|c|c|c|c|c|c|c|}
\hline \multirow{2}{*}{$\mathrm{hr}$} & \multirow[b]{2}{*}{ Unit 1} & \multirow[b]{2}{*}{ Unit 2} & \multirow[b]{2}{*}{ Unit 3} & \multicolumn{4}{|c|}{ Power output (MW) } & \multirow[b]{2}{*}{ Unit 8} & \multirow[b]{2}{*}{ Unit 9} & \multirow[b]{2}{*}{ Unit 10} & \multirow{2}{*}{$\begin{array}{l}\text { Total } \\
\text { power } \\
\text { (MW) }\end{array}$} & \multirow{2}{*}{$\begin{array}{c}\text { Cost } \\
(\$)\end{array}$} \\
\hline & & & & Unit 4 & Unit 5 & Unit 6 & Unit 7 & & & & & \\
\hline 1 & 455 & 295 & 0 & 0 & 0 & 0 & 0 & 0 & 0 & 0 & 750 & 14554.50 \\
\hline 2 & 455 & 345 & 0 & 0 & 0 & 0 & 0 & 0 & 0 & 0 & 800 & 15427.42 \\
\hline 3 & 455 & 395 & 0 & 0 & 0 & 0 & 0 & 0 & 0 & 0 & 850 & 16301.89 \\
\hline 4 & 455 & 365 & 0 & 130 & 0 & 0 & 0 & 0 & 0 & 0 & 950 & 18637.68 \\
\hline 5 & 455 & 415 & 0 & 130 & 0 & 0 & 0 & 0 & 0 & 0 & 1000 & 19512.77 \\
\hline 6 & 455 & 455 & 0 & 130 & 60 & 0 & 0 & 0 & 0 & 0 & 1100 & 21860.29 \\
\hline 7 & 455 & 455 & 0 & 130 & 110 & 0 & 0 & 0 & 0 & 0 & 1150 & 22879.12 \\
\hline 8 & 455 & 455 & 0 & 130 & 160 & 0 & 0 & 0 & 0 & 0 & 1200 & 23917.85 \\
\hline 9 & 455 & 455 & 130 & 130 & 130 & 0 & 0 & 0 & 0 & 0 & 1300 & 26184.02 \\
\hline 10 & 455 & 455 & 130 & 130 & 162 & 68 & 0 & 0 & 0 & 0 & 1400 & 28768.21 \\
\hline 11 & 455 & 455 & 130 & 130 & 162 & 80 & 0 & 13 & 0 & 0 & 1425 & 30045.64 \\
\hline 12 & 455 & 455 & 130 & 130 & 162 & 68 & 25 & 0 & 0 & 0 & 1425 & 29942.21 \\
\hline 13 & 455 & 455 & 130 & 130 & 162 & 0 & 68 & 0 & 0 & 0 & 1400 & 29221.58 \\
\hline 14 & 455 & 423 & 130 & 130 & 162 & 0 & 0 & 0 & 0 & 0 & 1300 & 26290.58 \\
\hline 15 & 455 & 455 & 130 & 0 & 160 & 0 & 0 & 0 & 0 & 0 & 1200 & 23948.99 \\
\hline 16 & 455 & 455 & 130 & 0 & 35 & 0 & 0 & 0 & 0 & 0 & 1075 & 21389.48 \\
\hline 17 & 455 & 455 & 130 & 0 & 25 & 0 & 0 & 0 & 0 & 10 & 1075 & 22138.16 \\
\hline 18 & 455 & 455 & 130 & 0 & 60 & 0 & 0 & 0 & 0 & 0 & 1100 & 21891.43 \\
\hline 19 & 455 & 455 & 130 & 0 & 135 & 25 & 0 & 0 & 0 & 0 & 1200 & 24358.09 \\
\hline 20 & 455 & 455 & 130 & 0 & 162 & 73.005 & 25 & 0 & 0 & 0 & 1300.005 & 27197.98 \\
\hline 21 & 455 & 455 & 130 & 0 & 162 & 80 & 0 & 0 & 17.995 & 0 & 1299.995 & 27351.86 \\
\hline 22 & 455 & 455 & 0 & 0 & 162 & 28 & 0 & 0 & 0 & 0 & 1100 & 22098.01 \\
\hline 23 & 455 & 315 & 0 & 130 & 0 & 0 & 0 & 0 & 0 & 0 & 900 & 17764.14 \\
\hline 24 & 455 & 215 & 0 & 130 & 0 & 0 & 0 & 0 & 0 & 0 & 800 & 16021.71 \\
\hline
\end{tabular}


TABLE 12

UNIT COMMITMENT RESULT OF SCENARIO 4

\begin{tabular}{|c|c|c|c|c|c|c|c|c|c|c|c|c|}
\hline \multirow{2}{*}{$\mathrm{hr}$} & \multirow[b]{2}{*}{ Unit 1} & \multirow[b]{2}{*}{ Unit 2} & \multirow[b]{2}{*}{ Unit 3} & \multicolumn{4}{|c|}{ Power output (MW) } & \multirow[b]{2}{*}{ Unit 8} & \multirow[b]{2}{*}{ Unit 9} & \multirow[b]{2}{*}{ Unit 10} & \multirow{2}{*}{$\begin{array}{l}\text { Total } \\
\text { power } \\
(\mathrm{MW})\end{array}$} & \multirow{2}{*}{$\begin{array}{c}\text { Cost } \\
(\$)\end{array}$} \\
\hline & & & & Unit 4 & Unit 5 & Unit 6 & Unit 7 & & & & & \\
\hline 1 & 455 & 320 & 0 & 0 & 0 & 0 & 0 & 0 & 0 & 0 & 775 & 14990.77 \\
\hline 2 & 455 & 370 & 0 & 0 & 0 & 0 & 0 & 0 & 0 & 0 & 825 & 15864.46 \\
\hline 3 & 455 & 395 & 0 & 0 & 0 & 0 & 0 & 0 & 0 & 0 & 850 & 16301.89 \\
\hline 4 & 455 & 365 & 0 & 130 & 0 & 0 & 0 & 0 & 0 & 0 & 950 & 18637.68 \\
\hline 5 & 455 & 415 & 0 & 130 & 0 & 0 & 0 & 0 & 0 & 0 & 1000 & 19512.77 \\
\hline 6 & 455 & 385 & 130 & 130 & 0 & 0 & 0 & 0 & 0 & 0 & 1100 & 21879.33 \\
\hline 7 & 455 & 435 & 130 & 130 & 0 & 0 & 0 & 0 & 0 & 0 & 1150 & 22755.04 \\
\hline 8 & 455 & 455 & 130 & 130 & 0 & 30 & 0 & 0 & 0 & 0 & 1200 & 24149.97 \\
\hline 9 & 455 & 455 & 130 & 130 & 130 & 0 & 0 & 0 & 0 & 0 & 1300 & 26184.02 \\
\hline 10 & 455 & 455 & 130 & 130 & 162 & 68 & 0 & 0 & 0 & 0 & 1400 & 28768.21 \\
\hline 11 & 455 & 455 & 130 & 130 & 162 & 58 & 0 & 10 & 0 & 0 & 1400 & 29456.25 \\
\hline 12 & 455 & 455 & 130 & 130 & 162 & 58 & 0 & 10 & 0 & 0 & 1400 & 29456.25 \\
\hline 13 & 455 & 455 & 130 & 130 & 162 & 0 & 68 & 0 & 0 & 0 & 1400 & 29221.58 \\
\hline 14 & 455 & 455 & 130 & 130 & 105 & 0 & 25 & 0 & 0 & 0 & 1300 & 26842.13 \\
\hline 15 & 455 & 455 & 130 & 0 & 135 & 0 & 25 & 0 & 0 & 0 & 1200 & 24601.13 \\
\hline 16 & 455 & 455 & 130 & 0 & 60 & 0 & 0 & 0 & 0 & 0 & 1100 & 21891.43 \\
\hline 17 & 455 & 455 & 130 & 0 & 60 & 0 & 0 & 0 & 0 & 0 & 1100 & 21891.43 \\
\hline 18 & 455 & 455 & 130 & 0 & 60.001 & 0 & 0 & 0 & 0 & 0 & 1100.001 & 21891.45 \\
\hline 19 & 455 & 455 & 130 & 0 & 160 & 0 & 0 & 0 & 0 & 0 & 1200 & 23948.99 \\
\hline 20 & 455 & 455 & 0 & 130 & 162 & 73 & 0 & 0 & 0 & 0 & 1275 & 25992.73 \\
\hline 21 & 455 & 455 & 0 & 130 & 162 & 73.995 & 0 & 0 & 0 & 0 & 1275.995 & 26015.92 \\
\hline 22 & 455 & 455 & 0 & 130 & 0 & 60 & 0 & 0 & 0 & 0 & 1100 & 21945.19 \\
\hline 23 & 455 & 315 & 0 & 130 & 0 & 0 & 0 & 0 & 0 & 0 & 900 & 17764.14 \\
\hline 24 & 455 & 215 & 0 & 130 & 0 & 0 & 0 & 0 & 0 & 0 & 800 & 16021.71 \\
\hline
\end{tabular}

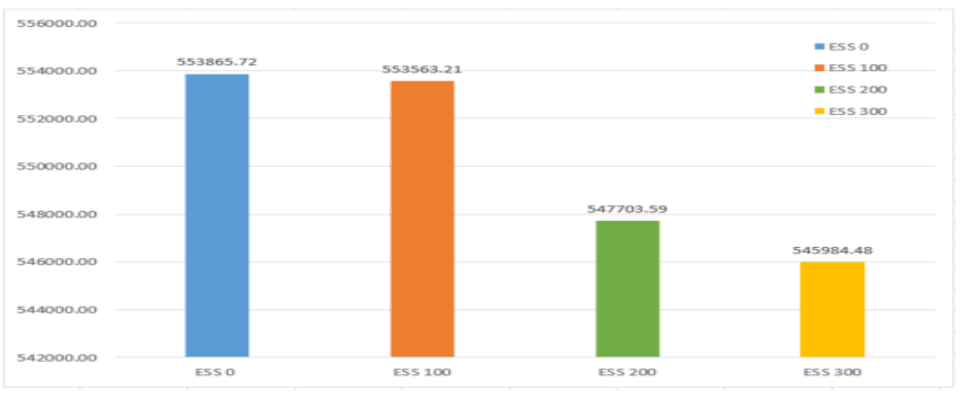

Fig. 3. Total cost of each scenario 
TABLE 13

POLLUTANTS EMISSIONS OF EACH SCENARIO (TON/DAY)

\begin{tabular}{cccc}
\hline \hline & CO2 & SOx & NOx \\
\hline case 1 & 5421.86 & 3.47 & 5.44 \\
case 2 & 5455.60 & 3.51 & 5.48 \\
case 3 & 5534.56 & 3.60 & 5.61 \\
case 4 & 5554.47 & 3.62 & 5.64 \\
\hline \hline
\end{tabular}

TABLE 14

POLLUTANTS TREATMENT COST OF EACH SCENARIO (TEN MILLION KRW)

\begin{tabular}{lcccc}
\hline \hline & CO2 & SOx & NOx & Total \\
\hline case 1 & 11.39 & 5.57 & 6.20 & 23.16 \\
case 2 & 11.46 & 5.64 & 6.25 & 23.35 \\
case 3 & 11.62 & 5.77 & 6.40 & 23.80 \\
case 4 & 11.66 & 5.80 & 6.43 & 23.90 \\
\hline \hline
\end{tabular}

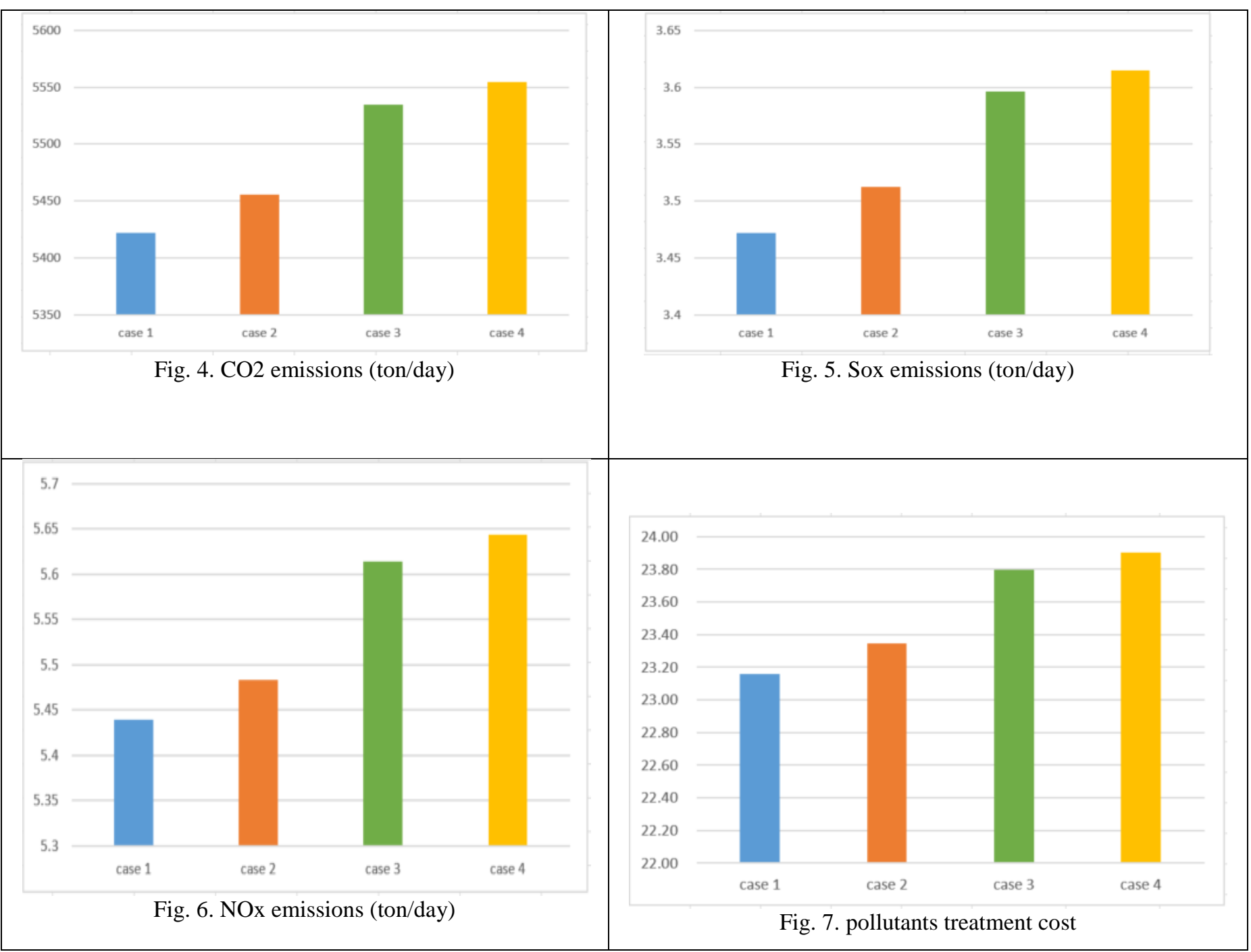

\section{CONCLUSION}

The object of this study is to research relationship between air pollutants emissions in power sector of Korea and capacity of BESS which is operated for maximizing customer's profits in the near future. It is observed from results that a gradual increasing total capacity of customer's ESS can reduce total cost in power system, but it increases pollutants emissions-NOx, SOx, CO2 and treatment cost. It was revealed that Spread of ESS for customer in the near future could have a negative effect in 
some way. The study was basic research using IEEE 39-bus data which was roughly adjusted to be similar but simplified for real power system of Korea. It requires a necessity for a further study in which many things are considered to be carried out.

\section{REFERENCES}

[1] Xu, P. Li and B. Zhao, "Optimal scheduling of microgrid with consideration of demand response in smart grid," in Networking, Sensing and Control (ICNSC), IEEE 12th International Conference on, IEEE , pp. 426431, 2015.

[2] L. Y. Kyeong, "Research issue, KEPCO economy \& Management Research Institute," KEMRI Power Economics Review, vol. 12, pp. 29-30, 2016.

[3] D. G. Kim, Y. S. Eom, J. H. Hong, S. J. Lee, S. K. Seok, D. G. Lee, E. J. Lee and S. A. Bang, "A study on the estimation of air pollutants emission factors in electric power plants, korean society for atmospheric

\section{ACKNOWLEDGEMENT}

This work is financially supported by Korea Ministry of Land, Infrastructure and Transport (MOLIT) as 「U-City Master and Doctor Course Grant Program.

environment," Journal of Korean Society for Atmospheric Environment, vol. 20, no. 3, pp. 281-290, 2010.

[4] Ministry of Trade, Industry and Energy, "The sixth basic plan on electricity demand and supply," 2013.

[5] J. W. Riekert and S. F. Koch, "Projecting the external health costs of a coal-fired power plant: The case of Kusile," Journal of Energy in Southern Africa, vol. 23, no. 4, pp. 52-66, 2012.

[6] P. Yong-Gi, B. Min-Kyu and R. Jae-Hyung, "A study of system fuel cost savings by use of the energy storage system," in The Korean Institute of Electrical Engineers Summer Conference, 2013, pp. 470-471.

— This article does not have any appendix. — 